Archives de sciences sociales des religions

176 | octobre-décembre 2016

Bulletin Bibliographique

\title{
Intransigeantisme-libéralisme-socialisme : le triangle interprétatif de Poulat en débats
}

Frédéric Gugelot

\section{(2) OpenEdition \\ Journals}

Édition électronique

URL : http://journals.openedition.org/assr/28117

DOI : $10.4000 /$ assr. 28117

ISSN : $1777-5825$

Éditeur

Éditions de l'EHESS

Édition imprimée

Date de publication : 31 décembre 2016

Pagination : 73-87

ISSN : 0335-5985

Référence électronique

Frédéric Gugelot, « Intransigeantisme-libéralisme-socialisme : le triangle interprétatif de Poulat en débats », Archives de sciences sociales des religions [En ligne], 176 | octobre-décembre 2016, mis en ligne le 01 janvier 2019, consulté le 03 janvier 2020. URL : http://journals.openedition.org/assr/28117 ; DOI : 10.4000/assr.28117

(C) Archives de sciences sociales des religions 


\title{
Frédéric Gugelot
}

\section{Intransigeantisme-libéralisme-socialisme : le triangle interprétatif de Poulat en débats}

\begin{abstract}
Qu'il est doux de sentir venir à soi des tendresses étrangères, quand on a été incompris et supplicié par les siens. Jules Vallès, L’Insurgé, Paris, Livre de Poche, 1972, p. 50.
\end{abstract}

Les premiers livres d'Émile Poulat, édition de sa thèse sur le modernisme et essais sur les prêtres ouvriers et sur l'intégrisme, ont suscité le débat dès leur parution. Mais les deux livres publiés coup sur coup en 1977, Catholicisme, démocratie et socialisme et Église contre bourgeoisie. Introduction au devenir $d u$ catholicisme actuel, ont soulevé une vive interpellation de son auteur, peutêtre parce que, à la différence des précédents, ces nouveaux ouvrages aspirent à faire système. Trois niveaux de réception permettent de dessiner les conditions de cette réception, ses thèmes, ses acteurs. Les premières lectures par les historiens de l'œuvre d'Émile Poulat se reflètent dans les comptes rendus et les évocations en séminaire. Jean-Marie Mayeur apparaît alors comme un précoce introducteur et débatteur des thèses du "sociologue » d'autant qu'elles entrent en résonance directe avec ses propres recherches ${ }^{1}$. Le premier lien entre les deux chercheurs est donc la proximité de leurs thèmes ${ }^{2}$. Tous deux s'intéressent tôt à la «tradition catholique sociale » que l'historien définit très justement comme "les écoles de pensée et les mouvements qui ont voulu résoudre la question sociale, c'est-à-dire l'ensemble des problèmes sociaux (et non pas seulement ouvriers) nés du libéralisme économique et de la révolution industrielle, à la lumière des enseignements du catholicisme $»^{3}$. Ce qui explique la participation

1. Je remercie vivement les enfants de Françoise et Jean-Marie Mayeur, et tout particulièrement Catherine Mayeur-Jaouen, de m'avoir autorisé à consulter les archives déposées aux Archives nationales.

2. Poulat rendra compte du livre de Mayeur sur la démocratie chrétienne en ces termes: "On attendait depuis longtemps ce livre qui n'en remplacera aucun parce que jamais encore n'avait été entrepris une étude d'ensemble de ce genre. Il ne décevra pas ", Archives de Sciences Sociales des Religions, vol. 50, 1980, $\mathrm{n}^{\circ}$ 2, p. 313.

3. J.-M. Mayeur, "Catholicisme intransigeant, catholicisme social, démocratie chrétienne ", Les Annales, mars-avril 1972, repris dans Catholicisme social et démocratie chrétienne. Principes romains, expériences françaises, Paris, Éditions du Cerf, 1986, p. 19, note 10. Il consacrera à Poulat un article particulier, "Autour de l'œuvre d'Émile Poulat ", repris dans le même ouvrage. 
de Poulat au jury de thèse de Mayeur. Il existe une vraie proximité intellectuelle entre les deux hommes dont témoigne une lettre du premier : "Vous êtes certainement, Madame Mayeur et vous, si je n'abuse pas - ceux où je retrouve le plus ma sensibilité dans l'analyse des phénomènes religieux contemporains ${ }^{4}$. " Mais d'autres au même moment le lisent et le reçoivent différemment, notamment ses " collègues" sociologues (Poulat est sociologue selon son recrutement au CNRS même si, sur le fond, lui-même hésite et ses collègues en doutent). Un troisième cercle de lecteurs entre alors en jeu, toute une frange militante de chrétiens, marquée à gauche. Ces trois milieux de réception, et donc d'interprétation, cernent la manière dont Poulat a été lu et ce qui a été retenu ou non de ses propositions à un moment où les recompositions intellectuelles et scientifiques sont importantes dans le contexte des suites du Concile Vatican II.

Les deux essais de 1977 sont tous deux édités chez Casterman ; la couverture $\mathrm{du}$ premier est bleue, celle du second, Église contre bourgeoisie, est rouge. L'auteur définit le second ainsi : "Ce n'est même pas un livre, avec la plénitude que comporte ce mot. C'est, plus simplement, l'introduction à un volume dont l'a fait détacher l'ampleur qu'ils (les “discours") en sont venus à prendre. Elle a donc reçu son autonomie, tout en restant ordonnée à ce travail plus technique et plus circonscrit qui, en fin de compte, l'aura précédée de quelques mois sur le marché : Catholicisme, Démocratie et Socialisme [...], lui-même faisant suite à Intégrisme et Catholicisme ${ }^{5}$."

Les deux livres défendent la même thèse. Mais de façon inversée, le second exprime la thèse que le premier démontre par l'exemple ${ }^{6}$. L'idée centrale est «le lieu commun si répandu en notre temps sur la collusion de l'Église et de la bourgeoisie masque la réalité d'un conflit entre l'une et l'autre ${ }^{7}$.

Un premier élément favorise la réception des propositions de Poulat par les historiens sensibles aux faits religieux : la méthode. À l'occasion du séminaire qu'il tient à l'Université Paris IV-Sorbonne, le 9 janvier 1981, Mayeur résume ces éléments de méthode. Celui-ci est un «chercheur remarquable » par son érudition et sa " pénétration psychologique des pensées religieuses ». L'usage des écrits et des textes, néanmoins plus que des archives, permet aux historiens de se sentir en terrain connu. Poulat ne cessait d'ailleurs d'affirmer que l'érudition manquait plus aux sociologues qu'aux historiens, ces derniers étant de surcroît, à ses yeux, plus proches des faits que les premiers. Pour lui, la conceptualisation

4. Lettre d'É. Poulat à J.-M. Mayeur du 30 juin 1986. Papiers J.-M. Mayeur, Archives nationales.

5. É. Poulat, Catholicisme, démocratie et socialisme, Paris, Casterman, 1977, p. 9.

6. «Le rapprochement des deux ouvrages [...] ne présente pas d'inconvénients [...] l'étude du cas de Benigni devant illustrer concrètement les thèses de l'autre ", J. Prévotat, Revue d'histoire de l'Église de France, vol. 64, no 173, 1978, p. 265.

7. J.-M. Mayeur, op. cit., p. 41. 
des sociologues était un bon moyen d'éviter l'érudition ${ }^{8}$. Ces éléments, Mayeur les avait déjà notés dans le compte rendu de trois livres de Poulat, les deux de 1977 et celui de 1980, Une Église ébranlée, publié par Les Annales de novembredécembre 1980. Il insiste sur "le souci de rigueur positive et d'érudition critique », et il ajoute : «É. Poulat apporte, souvent à partir de sources de première main, des vues neuves ${ }^{9}$. " On comprend que Mayeur s'y retrouve avec cet " historien des textes ${ }^{10}$, même si à aucun moment, le premier n'identifie le second à un historien à part entière. On pourrait reprendre l'heureuse formule de Dominique Dubarle pour définir l'ouvrage Église contre bourgeoisie : "Ce n'est pas vraiment une histoire, et pourtant c'est le travail d'un historien et qui met en œuvre bien des références historiques ${ }^{11}$."

Mais au-delà des proximités de méthode, ce sont les propositions de Poulat qui apparaissent opératoires pour les historiens. Sur le plan épistémologique, Mayeur note la distinction entre histoire des idées et histoire religieuse, tant cette dernière ne se réduit pas à une " histoire d'idéologies et de stratégies " ${ }^{12}$. Il partage avec Poulat l'idée si juste qu' "un système d'idées ne constitue jamais un monde clos ${ }^{13}$. Le compte rendu des Annales s'achève sur un projet : "Rechercher comment une structure idéologique se défait, puis se recompose, telle est la tâche essentielle : certaines composantes du système de pensée antérieur s'estompent, d'autres demeurent et prennent une autre signification, une autre perspective ${ }^{14}$. » C'est bien le couple infernal "continuité-changement » qui revient comme enjeu de cette histoire en train de se faire, ou de se défaire ${ }^{15}$. Ce qui néanmoins fait l'intérêt des historiens pour les publications de Poulat est un thème partagé et des résultats de recherche proches. D'autant que l'historien, Mayeur, comme le sociologue ont cherché à remettre dans une perspective plus longue et à relativiser les "nouveautés » et les présupposés idéologiques de leur temps.

8. Réflexion de Danièle Hervieu-Léger. «Débats du CéSor » consacré à la publication de la thèse d'É. Poulat, 14 janvier 2016. Elle avait répondu aux propositions de Poulat dans son ouvrage (avec F. Champion), Vers un nouveau christianisme?, Paris, Éditions du Cerf, 1987, p. 289-300.

9. J.-M. Mayeur, loc. cit.

10. Expression de Claude Langlois, avril 2016. Dans une lettre du 28 juin 1997 à C. Langlois, É. Poulat écrit sur l'appartenance à la sociologie : "Pour moi, c'est une question académique (...) Pour moi, claustrophobe, j'entends ne me fermer à rien de ce qui peut servir mes fins. Ceci dit, vous avez raison, de comparer des pratiques [souligné par l'auteur] ». Papiers C. Langlois. Discussion avec C. Langlois, avril 2016. Je le remercie de m’avoir reçu pour évoquer ses souvenirs.

11. Compte rendu du P. D. Dubarle, Revue des sciences philosophiques et théologiques, t. LXI, 1977, p. 654.

12. J.-M. Mayeur, op. cit., p. 43.

13. Ibidem.

14. Ibidem.

15. Voir d'un historien qui n'est pas du "groupe de Créteil ", Émile Goichot, "Penser le changement religieux. À propos d'ouvrages récents de M. É. Poulat ", Journal de psychologie, $\mathrm{n}^{\circ} 4,1977$, p. 471-482. 
Le débat se cristallise autour de la proposition de Poulat d'un triangle interprétatif du catholicisme que Mayeur explicite, dans la préface de 1986 à son recueil d'articles : "Nous allions à l'encontre des idées reçues en France qui faisaient tout bonnement du catholicisme social et de la démocratie chrétienne les héritages du catholicisme libéral ${ }^{16}$. " Si ces deux formes d'expression du catholicisme au sein de la modernité ne sont pas issues du catholicisme libéral, quelle est leur matrice?

Depuis 1970-1971, Jean-Marie Mayeur est professeur à la nouvelle Université de Paris XII-Créteil ${ }^{17}$. Il y organise un séminaire d'histoire religieuse d'une dizaine de séances par an " au cours desquelles ses membres ou des chercheurs invités présentent leurs travaux, tandis que Mayeur assure l'actualité de l'historiographie internationale, en particulier allemande ${ }^{18}$. Ce séminaire apparaît comme un moment important de redéfinition de l'objet religieux comme objet des sciences sociales. Il s'inscrit plus largement dans une évolution du champ : «Après une phase économiste, ce n'est que dans les années 1970 que ce secteur de recherche (l'histoire religieuse) connaît un véritable élan, profitant de la vogue pour l'histoire des mentalités ${ }^{19}$. " C'est dans ce cadre qu'à trois reprises, au moins, ces livres sont débattus.

Le 15 mars 1974, le thème du jour est le catholicisme intransigeant selon Poulat. Des notes prises par Étienne Fouilloux nous permettent de dégager les grandes lignes mises en avant lors de ces discussions. Pour éviter de puiser dans un «stock de catégories assez limité et polémique [souligné dans le texte] », et pour «aboutir à une typologie ", Poulat propose d'abord une chronologie: "Pour É. P., longue époque de crise à répétition [...] "De l'abbé Grégoire aux prêtres ouvriers" (art. ancien ${ }^{20}$ ) aujourd'hui “De Port-Royal à V II" ${ }^{21}$. " On

16. J.-M. Mayeur, op. cit., p. 12.

17. Émile Poulat le félicite: « Nous nous réjouissons fort avec vous de cette élection attendue ». Lettre du 23 juillet 1969, Papiers J.-M. Mayeur, Archives nationales.

18. G. Cuchet, D. Pelletier, "Claude Langlois et le "moment Créteil" de l'histoire religieuse contemporaine ", in Langlois C., Le continent théologique, Rennes, Presses universitaires de Rennes, 2016, p. 8-9.

19. C. Delacroix, F. Dosse, P. Garcia, Les courants historiques en France XIXe-XXe siècle, Paris, Gallimard, 2007, p. 464.

20. Il s'agit de "Modernisme" et "intégrisme". Du concept polémique à l'irénisme critique ", Archives de sociologie des religions, 27, 1969, p. 3-28, qui débute par "De l'abbé Grégoire aux prêtres-ouvriers ". Poulat achève son article sur cette question : « Comment catholicisme social et catholicisme intégral en vinrent-ils donc à s'opposer après n'avoir été qu'un ? (...) L'intransigeantisme catholique a éclaté : on ne peut que s'accuser de "modernisme" et d'“intégrisme" " (p. 27).

21. Notes prises par Étienne Fouilloux au cours de ces séminaires. Papiers É. Fouilloux. Je remercie vivement Étienne Fouilloux de m'avoir communiqué ses documents et pour ses réflexions sur ce thème. Claude Langlois se souvient avoir, à l'occasion d'un de ces séminaires, présenté aussi du Poulat. Il suivra les séminaires de celui-ci après 1968, réalisera à partir de 1972 des comptes rendus dans les Archives de Sciences Sociales des Religions où Poulat est responsable du Bulletin bibliographique. 
notera le glissement chronologique, qui est aussi une extension, et le passage de frontières cléricales à des seuils chronologiques. Les historiens présents ne peuvent pas être insensibles à cette double évolution, non seulement parce qu'elle répond à leur propre méthodologie, mais aussi parce qu'ils sont tous marqués, d'une façon ou d'une autre par le récent Concile Vatican II et qu'historiens, sociologues et militants s'interrogent sur les retombées de celui-ci. De plus, ils aspirent à un dépassement de l'histoire religieuse traditionnelle d'Église, incarnée par la Revue d'histoire de l'Église de France. En 1974, le groupe de Créteil est à l'origine de la fondation de l'Association française d'histoire religieuse contemporaine (AFHRC) dont le premier président est Mayeur ${ }^{22}$. Il est d'ailleurs frappant de voir que, sur ce point, ces historiens rejoignent les membres du Groupe de sociologie des religions (GSR) au CNRS ${ }^{23}$, dont Poulat, dans le refus de la subordination du travail sociologique à la perspective pastorale ${ }^{24}$. Autre point de proximité entre les deux champs, cette autonomisation face à des enjeux ecclésiaux. Ils participent d'un processus plus large de déconfessionnalisation qui se traduit par une pluralisation de perspectives. C'est un moment clé pour l'histoire religieuse contemporaine tant dans son détachement par rapport à une histoire de l'Église que dans sa reconnaissance institutionnelle propre au moment où, sous l'influence de Michel de Certeau ${ }^{25}$, le Groupe de La Bussière réoriente ses travaux en faveur d'une histoire plus culturelle du religieux.

Sur le fond, à travers l'étude de deux échecs, le modernisme et les prêtres ouvriers, Poulat identifie le « barrage » : ce n'est pas l'intégrisme, mais « le catholicisme intransigeant, modèle dominant de l'Égl. Cath. De 1850 à 1960, défini par le Syllabus ${ }^{26}$. Alors que le catholicisme libéral serait dans un schéma binaire, vieille Église/société moderne, science/foi, spirituel/temporel, le catholicisme intransigeant, dominant, s'inscrit au contraire dans un schéma ternaire : intransigeantisme/bourgeoisie/prolétariat. "Il est la matrice d'un ensemble de manifestations de ce qui était à l'origine l'action catholique : le catholicisme social, la démocratie chrétienne, le syndicalisme chrétien, dont le type doctrinal

22. Voir l'excellent documentaire de Frédéric Lunel, AFHRC 1974-2014. Quarante ans d'histoire, 2014. Poulat envoie ainsi à Claude Langlois son livre Église contre bourgeoisie avec cette dédicace : "Pour Claude Langlois, cette analyse non conformiste d'un phénomène paradoxal, en amicale sympathie ". Il préface aussi le livre d'un autre membre du groupe, Claude Savart, consacré à L'Abbé Jean-Hippolyte Michon 1806-1881, contribution à l'étude du libéralisme catholique au XIX ${ }^{e}$ siècle, publié en 1971 aux Belles Lettres.

23. P. Lassave, «Le groupe de sociologie des religions (1954-1995) : éléments de parcours d'un microcosme refondateur ", in Béraud C., Duriez B., Gasquet B. de, Actes du colloque AFSR sur le religieux dans les sciences sociales (2014), Rennes, Presses universitaires de Rennes, à paraître.

24. Voir l'article d'Olivier Chatelan, «Émile Poulat et les enquêtes sociographiques de pratique cultuelle (1956-1970) " dans ce même dossier.

25. Sur ce point, voir É. Fouilloux, M. Fourcade, Lignes de vie et de recherche, Paris, Éditions CLD, 2015, p. 117 et p. 129-130.

26. Notes d'É. Fouilloux, Papiers É. Fouilloux. 
est resté cohérent même quand il a donné lieu à des développements nouveaux ${ }^{27}$. " Mayeur résume l'idée dans un article du Monde, du 15-16 janvier 1978, intitulé «L'Église et la bourgeoisie »: " On lui sera également reconnaissant de substituer à une lecture dualiste de l'histoire des idéologies et des forces politiques dans l'Europe contemporaine qui, selon le cas français, privilégie le conflit de la droite et de la gauche, un schéma plus complexe. Celui-ci oppose, dans un jeu triangulaire, à partir de la fin du dix-neuvième siècle, l'expression politique du catholicisme, les libéraux et les socialistes ${ }^{28}$. " Il ajoute que "le modèle d'interprétation d'É. Poulat fait apparaître les virtualités contestataires de l'enseignement social et politique du christianisme ». Et c'est bien cette grille d'interprétation qui résonne dans les travaux des historiens présents et en particulier ceux de Mayeur.

Le 18 novembre 1977, le séminaire évoque les deux livres de Poulat, plus longuement le premier sur Benigni, plus rapidement le second. Cette différence de traitement illustre bien la réception décalée des deux ouvrages. Plusieurs " observations » sont émises. La première est de «surestimer " 29 la nouveauté et l'apport de sa thèse, les historiens citent les travaux antérieurs de Duroselle, Aubert, Touchard, Rémond, malgré sa conception souvent binaire droitegauche. "Si les catholiques se divisent entre droite et gauche, ce n'est pas d'abord pour des raisons politiques, mais pour des raisons religieuses qui tiennent à une certaine conception du christianisme et de sa fonction sociale et en correspondance avec une nuance de sensibilité religieuse, une forme de dévotion, voire même une théologie ${ }^{30}$. " Rémond en particulier est convaincu que l'Église est en train de sortir de l'intransigeantisme en conciliant les valeurs chrétiennes avec le monde moderne. "On y voit se heurter vivement deux catholicismes : l'un traditionnel, conservateur, lié à l'ordre établi, l'autre plus ouvert, à l'affût des innovations et qui prend ses distances par rapport aux alliances traditionnelles $^{31}$. » Mayeur ajoutera le nom de Joseph Hours dans le compte rendu des Annales. Ceux-ci montraient déjà "fortement les origines intransigeantes du catholicisme social et de la démocratie chrétienne, et que celle-ci ne naissait pas du catholicisme libéral » ${ }^{32}$. Mayeur avait lui-même tôt noté que cette philosophie politique et sociale d'inspiration intransigeante avait eu une large influence sur le monde catholique : "On y souscrit d'autant plus volontiers qu'on a développé des thèmes du même ordre ${ }^{33}$. " Les historiens reçoivent bien l'idée du triangle catholicisme intransigeant-libéralisme-socialisme, passage de «deux à trois - il

27. "Émile Poulat. Église et Bourgeoisie ", compte rendu dactylographié de la réunion du Centre régional interuniversitaire d'histoire religieuse de Lyon du 3 mars 1976, p. 1.

28. J.-M. Mayeur, "L'Église et la bourgeoisie ", Le Monde, 15-16 janvier 1978.

29. J.-M. Mayeur, op. cit., p. 42.

30. R. Rémond, Vivre notre histoire, Paris, Le Centurion, 1976, p. 211.

31. Ibid., p. 212.

32. J.-M. Mayeur, op. cit., p 42.

33. Ibidem. 
a raison - avec tous les types de combinaison ", note Fouilloux ${ }^{34}$. Les historiens s'interrogent néanmoins sur la valeur de la notion de catholicisme libéral - catholicisme bourgeois, "ceux qui en prennent et en laissent ».

Si l'adoption du triangle interprétatif se fait sans grande difficulté, des critiques surgissent. La typologie ne leur apparaît pas assez rigoureuse surtout dans le passage de la « micro-histoire » à la " généralisation ». En particulier, le thème de l'innovation-changement ne serait pas suffisamment expliqué : "Comment une structure peut bouger et modifie chacun des éléments ${ }^{35}$ » ? Ces éléments rejoignent ceux de Roger Aubert dans la conclusion de son compte rendu : "Serais-je tenté de penser avec J.-M. Mayeur que "comme toute interprétation qui insiste sur la permanence des structures, celle-ci ne rend pas suffisamment compte des mutations et du changement" ${ }^{36}$. " Ce reproche est récurrent chez bien d'autres et sera partagé avec les sociologues. Au point que, lors du séminaire, on s'interroge : "On glisse à une histoire des idées pour elle-même " où « il [Poulat] durcit la continuité ${ }^{37}$. »

Après son élection à la Sorbonne, Mayeur poursuit le séminaire, maintenant commun avec celui de Créteil, et consacre le 9 janvier 1981 une nouvelle séance aux apports de Poulat autour du recueil d'articles que celui-ci vient de faire paraître ${ }^{38}$. Après une évocation de son cheminement, dont une thèse de théologie sur le modernisme à Fribourg, qui n'est donc pas si inconnue que cela à ce moment-là ${ }^{39}$, Mayeur articule les recherches de Poulot selon trois axes : socialismes utopiques/modernisme/prêtres ouvriers. Mayeur dégage deux thèmes dans les travaux de Poulat : passage du changement aux permanences, l'emprise croissante du modèle intransigeant, et des interrogations sur l'élimination du catholicisme éclairé et l'absence du catholicisme " privé ». Rien de nouveau donc dans la réception, la grille d'interprétation est faite et avancées et critiques rebondissent d'un livre à l'autre.

La réception des ouvrages de Poulat est donc réelle avec une lecture attentive de l'œuvre. La proximité se fonde d'abord sur des interrogations communes, des débats partagés et des méthodes croisées. Le livre bleu est, tant pour les historiens que pour les sociologues, on le verra, l'ouvrage de référence. Le rouge est beaucoup plus contesté. L'ambition n'est pas la même. Poulat le reconnaît en mars

34. Il reprendra ses réflexions avec des critiques rejoignant celles exprimées par le groupe dans un compte rendu intitulé "Du catholicisme intransigeant selon Émile Poulat ", Esprit, avril 1978, p. 116-121.

35. Notes d'É. Fouilloux, Papiers É. Fouilloux.

36. R. Aubert, Revue d'Histoire Ecclésiastique, t. LXXV, 1980, nº 1, p. 145.

37. Notes d'É. Fouilloux, Papiers É. Fouilloux. 1980.

38. É. Poulat, Changement, conflit et continuité de Pie XII à Jean-Paul II, Paris, Casterman,

39. F. Trémolières, "Introduction ", in Poulat É., Le désir de voir Dieu et sa signification dans la théologie française contemporaine, Paris, Desclée de Brouwer, 2015, p. 11. 
1976 : «Il le fait, non par volonté particulière pour la théorie, mais parce qu'il est arrivé à un moment de ses travaux où il éprouve le besoin de réfléchir à ce qui a été accumulé et de tenter une synthèse ${ }^{40}$. " C'est cette dernière tentative qui se heurte à une réception difficile surtout chez ses «collègues".

Poulat est d'autant plus sensible à cette réception chaleureuse par ces quelques historiens que du côté des sociologues la douche est froide. Deux comptes rendus en témoignent, celui de François-André Isambert et celui de Paul Ladrière. Il faut noter que les deux auteurs transmettront leurs articles à Mayeur, le premier avec la dédicace suivante, "À Jean-Marie Mayeur, en cordial hommage » et le dernier avec la suivante : «À Jean-Marie Mayeur, cette incursion dans son domaine ».

Isambert, lui-même membre avec Poulat du premier cercle des fondateurs du GSR, signe un article dans la Revue française de sociologie qui dès son titre illustre le refus d'une continuité : «Du Syllabus à Vatican II, ou les avatars de l'intransigeantisme ». Il insiste sur le second tome : "Le second tente de généraliser et d'actualiser tout à la fois, faisant du "catholicisme intransigeant" un modèle dont l'application permet, pense l'auteur, d'éclairer le "devenir du catholicisme actuel" ${ }^{41}$. » Le sociologue reconnaît l'apport du premier livre et «l'unité de position de Benigni à travers ses variations apparentes ${ }^{42}$. La position matricielle de l'intransigeantisme est acquise tant chez les historiens que chez les sociologues. Mais à la question de la « logique » ou de «l'identité » de l'intransigeantisme, le sociologue ne suit plus Poulat même s'il accepte l'idée que «la "permanence des institutions" [...] est un point capital qui fixe sans doute ce que peut avoir de plus solide cette notion d'intransigeantisme dont l'auteur veut faire un large usage " ${ }^{43}$. «Il y a, nous dit Émile Poulat, un modèle intransigeant. [...] Or autant l'intransigeantisme est apparu comme un concept historique heureux [...] pour éclairer la succession Pie IX-Léon XIII-Pie X, autant l'auteur semble avoir du mal à le préciser en tant que modèle sinon permanent, du moins récurrent ${ }^{44}$. L'intransigeantisme n'est pas un idéal-type : «Dira-t-on appliquée à l'Église catholique, l'intransigeantisme reviendrait à une conception de l'Église comme macro-secte $?^{45}$ » Le procès en vient à critiquer le titre même du deuxième ouvrage, décidément celui qui pose le plus de difficulté, Église contre bourgeoisie. Pour Isambert, il y est plus question de libéralisme que de bourgeoisie; il ne s'agit pas pour lui de " prise de positions antagonistes par rapport à des classes [...], mais par rapport à des "puissances" : les puissances de la richesse, de la

40. «Émile Poulat. Église et Bourgeoisie », compte rendu cit., p. 1.

41. F.-A. Isambert, "Du Syllabus à Vatican II, ou les avatars de l'intransigeantisme", Revue française de sociologie, no 4, octobre-décembre 1978, p. 603.

42. Ibid., p. 606 sq.

43. Ibid., p. 609.

44. Ibid., p. 610.

45. Ibid., p. 610 sq. 
politique des États, des organisations secrètes (franc-maçonnerie), des religions non-chrétiennes (judaïsme) » ${ }^{46}$. Impitoyable, le sociologue conclut en affirmant qu' " au mieux, il peut fonctionner en tant que type historique, comme l'éthique protestante de Weber ${ }^{47}$.

Paul Ladrière reconnaît aussi les apports de Poulat: "Origine intransigeante du mouvement social catholique, son antilibéralisme foncier, son objectif populaire ${ }^{48}$. " Mais « tout se complique quand É. Poulat introduit son schéma "triangulaire" ", selon lequel «le catholicisme intransigeant [...] posséderait en lui-même un tel degré de spécificité qu'il constituerait un troisième pôle ${ }^{49}$. Pour lui, " ce catholicisme intransigeant est contre-révolutionnaire [...]. C’est parce que contre-révolutionnaires que les intransigeants sont antilibéraux et antisocialistes ${ }^{50}$. " Et « le catholicisme intransigeant, parce qu'il est contrerévolutionnaire, ne se veut pas un catholicisme du peuple, mais un catholicisme pour le peuple " ${ }^{51}$. Mais le vrai problème est que Poulat "élève ce catholicisme intransigeant en principe explicatif du catholicisme actuel » ${ }^{52}$. Il ajoute : "Pour constituer le catholicisme intransigeant en modèle, malgré cette pression constante de l'histoire, É. Poulat est obligé de figer le catholicisme, le libéralisme et le socialisme [...] en entités modulables à volonté, mais chimiquement pures $^{53}$. " Il conclut : «Tous ces phénomènes n'expriment qu'une certaine, une relative continuité. Ils ne sont pas expliqués par elle. Ils sont aussi le lieu d'authentiques changements qui s'accomplissent dans des bouleversements dont l'effet est moins le désordre qu'une déstructuration-restructuration du champ. [...] La minutie patiente de l'explication du passé justifie-t-elle la hauteur avec laquelle est traité le présent ? $^{54}$ » Ladrière rejette l'équivalence entre intransigeantismelibéralisme-socialisme.

Si historiens et sociologues sont d'accord pour faire de l'intransigeantisme une alternative au monde moderne, devenue dominante dans le catholicisme, les premiers reçoivent le triangle que les seconds refusent; les deux néanmoins expriment un net refus de faire de l'intransigeantisme le seul modèle justifiant le tout du catholicisme. Seul, chez les sociologues, le dominicain Serge Bonnet défend les thèses de Poulat. Mais au-delà d'une certaine solidarité de " clercs ", il est aussi un marginal, qui a trouvé en Poulat un défenseur dans le débat sur

46. Ibid., p. 611.

47. Ibid., p. 612.

48. P. Ladrière, "L'intransigeance des origines et le devenir du catholicisme actuel ", Cahiers internationaux de sociologie, vol. LXVII, 1979, p. 298.

49. Ibid., p. 300.

50. Ibid., p. 302.

51. Ibid., p. 305.

52. Ibid., p. 308.

53. Ibid., p 309.

54. Ibid., p. 310 sq. 
la « religion populaire " au même moment ${ }^{55}$. Bonnet est favorable à «l'abandon de la bi-partition politico-religieuse ${ }^{56}$. Il précise : « L'intransigeantisme semble aujourd'hui négligeable. Mais, sous d'autres formes et avec d'autres couleurs que jadis, il demeure un constitutif (fondamental ? ultime ?) du catholicisme. Peut-il en être autrement ? Une Église qui ne veut pas se dissoudre dans le libéralisme ou le socialisme peut-elle récuser l'intégralisme ? ${ }^{57}$ » Quant à l'articulation continuité/changement, Bonnet propose de désigner la sociologie de Poulat comme « une sociologie de la permanence ou, pour être plus précis, de la moyenne durée ${ }^{58}$. Pirouette qui permet de tenir le modèle sans estomper les changements.

Au-delà de la réception scientifique, un troisième cercle de lecteurs illustre les difficultés rencontrées par les propositions de Poulat. Alors que le livre bleu est très bien accueilli, le rouge entraine de nombreuses réticences. Ce d'autant que les deux livres résonnent dans les débats qui agitent au même moment le catholicisme à la suite du Concile et de ses conséquences : le monde catholique, peut-il changer ? Et jusqu'à quel point ? Dès 1974, Michel de Certeau et JeanMarie Domenach parlent d'un "christianisme éclaté » ${ }^{59}$, tandis que Poulat consacre la première sous-partie du petit livre rouge à « Du changement religieux » : "Les catholiques étaient à droite. Voilà qu'ils changent de camp : ils sont en train de passer à gauche, et même à l'extrême gauche, brusquement à la suite d'une illumination ${ }^{60}$. Cette " conversion », Poulat en explicite l'enjeu dès l'introduction de ce discuté second volume: "À cheval sur la période moderne et contemporaine, le catholicisme de la Contre-Réforme - quatre siècles - avait été un temps de certitudes et de sécurité. [...] Il est [...] un fait [...] : le retournement de situation qui, ces dernières années, a introduit dans le mouvement catholique une véritable discontinuité ${ }^{61}$. » Il reconnaît donc une évolution, mais en rappelant que le christianisme social n'est pas issu du christianisme libéral, mais

55. Lettre d'É. Poulat à J.-M. Mayeur du 16 mars 1976, Papiers Mayeur, Archives nationales. "J'ai lu avec plaisir votre chronique du Monde. Merci personnel pour l'attention donnée à S. Bonnet et à ses travaux, jusqu'ici bien ignorés de la presse parisienne ". Pour une mise en contexte, voir É. Fouilloux, Les chrétiens français entre guerre d'Algérie et mai 1968, Paris, Parole et Silence, 2008, p. 305-323 et l'article de Yann Raison du Cleuziou, "Serge Bonnet et la sociologie de la domination cléricale. La controverse sur le catholicisme populaire ", Archives de Sciences Sociales des Religions, octobre-décembre 2016.

56. S. Bonnet, »Politique et religion dans l'œuvre d'Émile Poulat", Revue française de science politique, $\mathrm{n}^{\circ}$ 3, juin 1980, p. 600.

57. Ibid., p. 603.

58. Ibidem.

59. M. de Certeau, J.-M. Domenach, Le christianisme éclaté, Paris, Éditions du Seuil, 1974. Sur un autre «marginal» et sa réception chez les historiens à l'occasion de la polémique sur la «culture populaire », voir Denis Pelletier, «Loudun, Certeau, Mandrou. Un jésuite chez les historiens ", in Fouilloux É., Gugelot F. (dir.), Jésuites français et sciences humaines (Années 1960), Lyon, Chrétiens et sociétés, 2014, p. 157-173.

60. É. Poulat, Église contre bourgeoisie, op. cit., p. 14.

61. Ibid., p. 13. 
bien de la veine intransigeante. Or la fin des années 1970 apparaît comme un premier temps de bilan postconciliaire en particulier pour les chrétiens de gauche. Où en est-on de la tentative réformatrice du Concile ? Est-on dans une rupture instauratrice ? Certeau acte la fin de la tentative ${ }^{62}$. Plusieurs publications en témoignent. En 1975, Cité Nouvelle avait publié un dossier, dans plusieurs numéros, "Pour une histoire des chrétiens de gauche ». Esprit publie un numéro spécial en avril-mai 1977 sur les "militants d'origine chrétienne ». Certeau y publie un texte qui livre sa vision d'une dissémination de l'expérience spirituelle en contraste avec la situation antérieure : "Autrefois une Église organisait un sol, c'est-à-dire une terre constituée : à son intérieur, on avait la garantie sociale et culturelle d'habiter un champ de vérité ${ }^{63}$. " La revue Autrement de février de la même année évoque "À gauche, ces chrétiens ». René Rémond y note : "Ils [les chrétiens de gauche] inclinent à reprendre à leur compte l'idée que l'Église est un appareil qui a partie liée avec l'idéologie dominante et qui sert sans partage les intérêts de la bourgeoisie ${ }^{64}$. » Ce que remet en cause le schéma triangulaire. Des textes, des conférences et des réunions sont alors consacrés aux livres de Poulat au sein des chrétiens de gauche qui donne une actualité aux thèses de l'auteur.

Un groupe de travail « histoire des chrétiens " a été fondé à la revue Lettre en octobre 1975. Pour mener «la lutte idéologique sur le front religieux » ${ }^{65}$, il faut opposer " au discours dominant un discours alternatif, fondé à la fois sur une pratique sociale et sur une critique théorique alimentée par les sciences humaines " ${ }^{66}$. À la tradition magistérielle, ils opposent la mémoire retrouvée des dissidents, des exclus. Mais les spécialistes (Baubérot, Certeau, Fouilloux...) refusent d'abandonner les exigences de la méthode historique ${ }^{67}$, alors que le dominicain Antoine Lion n'hésite pas à leur demander de travailler « dans une perspective militante ", non de " récupérer dans quelques thèses destinées à grossir la masse des connaissances de l'institution universitaire " ${ }^{68}$. Dans le numéro 231

62. M. de Certeau, "L’Église catholique : la fin de la période postconciliaire », Universalia, 1977, p. 141-144.

63. M. de Certeau, "La faiblesse de croire", repris dans La faiblesse de croire, Paris, Éditions du Seuil, 1987, p. 307.

64. R. Rémond, "La greffe du marxisme et ses fruits ", in "À gauche, ces chrétiens ", Autrement, no 8, février 1977, p. 190.

65. J. Chatagner, "Après la rencontre des chrétiens marxistes à Chevilly-Larue. Un objectif et une stratégie ", Lettre, no 196, décembre 1974, p. 3.

66. Y. Tranvouez, "Les idées du ciel ne tombent pas juste ", in Pelletier D., Schlegel J.-L. (dir.), À la gauche du Christ, Paris, Éditions du Seuil, 2012, p. 527.

67. «Certains autres [historiens], sous l'influence croissante de Michel de Certeau, valorisaient au contraire la médiation de l'opération historiographique ", É. Fouilloux, M. Fourcade, op. cit., p. 131.

68. A. Lion, «Histoire de l'Église ou histoire des chrétiens ? Pistes pour une mise à jour de la face cachée de l'histoire du christianisme ", Lettre, no 224, avril 1977, p. 25-29. Cité par Y. Tranvouez, «Les idées du ciel ne tombent pas juste », art. cit., p. 528. 
de Lettre, intitulé « Nous, chrétiens de gauche », paraît un encadré signé de deux initiales M. F., anonyme, au titre énigmatique "Triangle ou trapèze " ${ }^{69}$. Ce texte appartient à un dossier plus large comprenant entre autre un texte d'Yvon Tranvouez, "jugé trop favorable » aux propositions de Poulat, avec qui il fait sa thèse ${ }^{70}$. Il est alors "flanqué " de " témoignages "militants" " ${ }^{71}$. Cet encadré est très critique : "Pour bien étudier son triangle, Monsieur Poulat semble en gratter les adhérences à tout ce qui l'enracine socialement. Il voit clairement [...] la spécificité irréductible du champ religieux, où les affrontements ne sont pas des reflets directs de conflits sociaux. Mais à couper ces débats de ce qui les fonde, et qu'ils traduisent et déplacent dans leur logique propre, on se prive de pouvoir comprendre, on se cantonne plus ou moins dans la confrontation des idées au sein du petit monde des hommes d'Église. Cette réduction [...] invalide une théorie globale ${ }^{72}$.»

Ce qui frappe est la proximité des reproches avec ceux des sociologues, surtout de Paul Ladrière: découplage social, enfermement dans une histoire des idées, critique que certains historiens évoquent aussi alors. Poulat réagit mal à cet encadré et cherche sans succès à en connaître l'auteur. Il envoie quatre lettres, au ton plus ou moins vif, à Jacques Chatagner. Celle du 7 décembre 1977 dénonce : "C'est ce même M. F. [...] et ceux qu'il représente que je soupçonne d'anarchisme historique et d'opération politique. C'est délibérément que j'ai adopté avec lui (ou elle) ce ton provocateur : en retour de ses insinuations gratuites, je lui envoie les miennes, qui le sont peut-être tout autant ${ }^{73}$. " En novembre 1977, Chatagner refuse de lever l'anonymat de l'auteur et de publier la réponse de Poulat, intitulé « Le pavé et la barricade ». Dans celui-ci, il affirme : « J'avais toujours perçu la Lettre et ses lecteurs comme le carrefour d'une diversité assez hétérogène : je découvre qu'elle a une ligne et que, comme la loi, nul n'est censé l'ignorer ${ }^{74}$. » Le reproche essentiel fait au chercheur serait donc de " "couper ces débats de ce qui les fonde", sans souci de leurs enracinements sociaux ». L'histoire faite par Poulat n'est en effet pas militante : "Si l'histoire que je fais est dénuée de toute valeur théorique et ne peut que dévier les militants chrétiens de leur chemin, par quelle autre histoire la remplacer ? Si je comprends

69. L'auteur en est le dominicain Antoine Lion, qui l'avouera à É. Fouilloux bien des années plus tard. La rédaction de la Lettre, souhaitant qu'un texte rejetant le schéma de Poulat soit publié, a essuyé de nombreux refus avant que le dominicain accepte de façon anonyme.

70. Y. Tranvouez, "Le passé politique de Dieu », Lettre, n 231, novembre 1977, p. 21-24.

71. Lettre d'É. Fouilloux à Jean Lebrun du 18 novembre 1977. Papiers É. Fouilloux.

72. M. F., "Triangle ou trapèze ?", Lettre, n 231, novembre 1977, p. 23.

73. Lettre d'Émile Poulat à Jacques Chatagner du 7 décembre 1977. Papiers É. Fouilloux. Poulat a souvent transmis les éléments à É. Fouilloux et J. Baubérot, membres du groupe « Histoire des chrétiens " à la Lettre où ils défendent la logique historique sur la logique militante.

74. É. Poulat, "Le pavé et la barricade », texte dactylographié, p. 1. Papiers É. Fouilloux. Poulat a fait parvenir ce texte à Fouilloux et Baubérot, conscient que les deux hommes soutiennent sa démarche scientifique. 
bien [...] par une histoire en souscription, l'histoire des hommes sans nom, celle qu'ignorent livres d'histoire et calendriers d'Église, celle qu'on a volée au peuple et dont il a besoin pour ses luttes d'aujourd'hui ${ }^{75}$. " Il rejette donc toute idée d'une écriture militante de l'histoire, mais ne répond pas aux critiques de fond. Jean Baubérot et Étienne Fouilloux s'opposent également à cette fabrique militante de l'histoire. Fouilloux livre au journaliste et historien Jean Lebrun une explication de cette difficile réception : "Le terrain devient de plus en plus chaud. Assez mécontente de se voir décortiquée de l'extérieur, la "gauche chrétienne" part à la recherche de ses ancêtres et son dualisme "bons progressistes crossés" - "méchants conservateurs" (je caricature !) se heurte de front au révisionnisme de Poulat précédé et suivi pour une bonne partie des historiens (Aubert, Mayeur... Tranvouez et moi-même). Quand les “chrétiens de gauche” reconnaissent leurs origines intransigeantes, c'est pour mieux insister sur les ruptures qui les en ont coupés ${ }^{76}$. » On comprend combien le triangle de Poulat ne répondait pas à cette attente.

En janvier 1978, Masses ouvrières, la revue de l'Action catholique ouvrière publie un texte commandé à Jean Lebrun ${ }^{77}$. Il s'ouvre sur un constat : "Quelquesuns parlent de catholicisme pluriel, de christianisme sans idéologie et nous croyons être parvenus au terme, notre espoir, pensons-nous, est réalisé. Enfin, la catholicité accepte les différences, on peut vivre autrement sa foi selon qu'on est dans l'horizon bouddhiste, dans l'horizon marxiste, etc. ${ }^{78}$ » On retrouve cette idée que l'étape post-conciliaire est bien celle d'un pluralisme catholique. Il ajoute : "La perversion du christianisme en un modèle totalisant, clôturant, la capture du message de libération chrétienne par l'idéologie, la dégénérescence de la vie, cela serait-il fini ? ${ }^{79}$ " Il s'interroge donc sur la disparition de l'intransigeantisme. Lebrun use alors de l'ouvrage de Poulat pour affirmer en conclusion que les « trois attitudes » existent toujours, distinguant ce qu'il appelle les « intégristes ", qui " défendent avant tout l'union indissociable entre Bible et tradition ", "les fondamentalistes ", " aussi peu portés à la spéculation qu'à la mystique, dévoués à l'institution et rassurés par elle » et les exégètes « libéraux " "depuis longtemps dans l'ère de la "modernistique", comme dit Poulat » ${ }^{80}$. Il conclut : "Étrange destin du langage catholique à vocation unifiante et universelle, devenu forêt de contradictions ${ }^{81}$. " Il reconnaît donc "le type historique " de l'intransigeantisme, mais en conclut que le modèle n'existe plus, la pluralité l'ayant remis profondément en cause.

75. Ibid., p. 2.

76. Lettre d'É. Fouilloux à J. Lebrun du 18 novembre 1977, Papiers É. Fouilloux.

77. Lettre de J. Lebrun à É. Fouilloux du 23 novembre 1977, Papiers É. Fouilloux.

78. J. Lebrun, "Mgr Umberto Benigni (1862-1934). Entre le libéralisme et le socialisme ", Masses ouvrières, janvier 1978, p. 14.

79. Ibid., p. 16.

80. Ibid., p. 28.

81. Ibid., p. 29. 
Il est intéressant de noter que le second livre passionne plus à l'extérieur du monde universitaire que le premier. Fouilloux propose alors à Lebrun de recevoir Poulat à l'antenne, pour rompre avec le silence de la grande presse sur ses derniers essais. Comme on le voit, les historiens jouent un rôle important pour la diffusion des idées du chercheur, Mayeur et Le Monde, Fouilloux et la radio. Le samedi 10 décembre 1977, l'enregistrement de l'émission «Les Lundis de l'Histoire " sur France Inter est consacré aux livres de Poulat, invité en même temps que Jacques Maître et Paul Ladrière. Les objections apportées aux livres pendant la discussion portent à nouveau sur la distinction structure/conjoncture, continuité/changement. L'émission est diffusée le 9 janvier 1978. Confrontations, le prolongement du Centre Catholiques des Intellectuels Français, organise le lundi 8 mai 1978, à 20 h 45 un débat autour d'Église contre bourgeoisie. C'est une fois de plus le second tome qui intéresse. Le texte d'invitation explicite les enjeux du débat : " Livre d'historien ${ }^{82}$ [...] il manifeste l'importance de la relation triangulaire qui, à la fin du XIX siècle, s'établit entre catholicisme, libéralisme et socialisme, et à la complexité des alliances qu'elle permet. Du coup, le premier, sous sa forme officielle au moins et par-delà la diversité des tendances qui l'habitent, retrouve une cohérence que nous avions oubliée. Oubli lourd de graves conséquences; car, à bien des égards, on y discerne l'origine du grand trouble qui s'empare de l'Église après Vatican II. Mais cette relation triangulaire elle-même n'est qu'une des formes du grand affrontement qui oppose l'Église et la Modernité. [...] “Tu as vaincu, Modernité”, énonce Émile Poulat en terminant son livre. Mais il faut aller plus loin et ajouter au constat de nouvelles questions. "Tu as vaincu. Soit, mais qu'as-tu vaincu ? Est-ce le catholicisme ? Ou ce catholicisme qui, sous des figures remarquablement différentes et renaissantes, s'était affronté à toi ?" 83 "Une fois de plus, si la grille de lecture intransigeante est acceptée, le modèle est rejeté. La difficile réception de l'œuvre de Poulat en 19771978 tient à cette division en deux ouvrages. Le livre bleu est reçu et le schéma triangulaire, limité chronologiquement du Syllabus au Concile, est reconnu. Le livre rouge est contesté tant sur le plan scientifique que militant. L'ambition théorique est acceptée par les historiens qu'elle conforte dans leurs propres recherches, mais rejetée au sein des sociologues. Poulat a conscience de l'impact immédiat de ses livres et des résistances qu'ils rencontrent. Dans une lettre à Fouilloux du 17 décembre 1977, il reconnaît : "Le devenir du catholicisme actuel ne serait-il qu'un éternel recommencement? Le déduire de mes travaux est de fait possible [...], mais à condition de se situer dans une démarche qui n'est pas la mienne. Ma question réelle est celle-ci : à quelle(s) condition(s) estil possible d'échapper au manège au lieu de seulement essayer tous les chevaux du manège sans changer les cavaliers ? $^{84}$ "

82. Certains n’hésitent donc pas à sauter le pas.

83. Tract de "Confrontations. Société, culture et foi ». Papiers É. Fouilloux. Voir sur le CCIF, C. Toupin-Guyot, Les intellectuels catholiques dans la société française, Rennes, Presses universitaires de Rennes, 2002.

84. Lettre d’É. Poulat à É. Fouilloux, 17 décembre 1977. Papiers É. Fouilloux. 
Grâce à une commune vision épistémologique et surtout une réelle convergence des recherches, les historiens sensibles aux questions d'histoire religieuse accueillent très bien le premier livre de 1977 sur Monseigneur Benigni. Ce travail illustre certainement pour eux les possibilités d'une histoire religieuse renouvelée et détachée. Du côté des sociologues, le projet de socio-histoire, est également salué, mais la tentative de modélisation qu'il vise manque pour eux de rigueur. Le livre rouge devient l'enjeu de débats entre collègues et franchit les frontières de la discussion scientifique. Disputé, il apparaît comme le révélateur d'un enjeu plus large du moment de sa parution, les interrogations sur les changements, possibles ou impossibles, au sein du catholicisme post-conciliaire. Chez les militants chrétiens de gauche, une résistance s'exprime contre le schéma triangulaire. Cette difficile réception contribue certainement à accentuer le sentiment d'isolement de Poulat.

Celui-ci en tire les leçons dans une lettre à Fouilloux : «La frontière entre historiens et sociologues n'est pas insurmontable, mais elle n'est pas seulement académique. Il y a vingt-cinq ans, je la croyais facile à abattre, et je crois avoir beaucoup essayé pour cela. Échec. [...] Je continue seulement à croire à l'amitié et aux relations entre gens de bonne volonté ${ }^{85}$. " Il est assez facile de reconnaître, parmi d'autres, sa relation avec Mayeur, à la fois introducteur des propositions du chercheur chez les historiens, mais aussi débatteur amical. Au-delà d'une réception critique, une réflexion partagée et un soutien réciproque qui n'empêche pas des divergences, il existe en effet une réelle attention de l'un envers l'autre dont témoigne leur correspondance.

\author{
Frédéric Gugelot \\ CERHIC-Reims et CéSor-EHESS \\ frederic.gugelot@univ-reims.fr
}

85. Lettre d'É. Poulat à É. Fouilloux, 3 février 1979. Papiers É. Fouilloux. 
